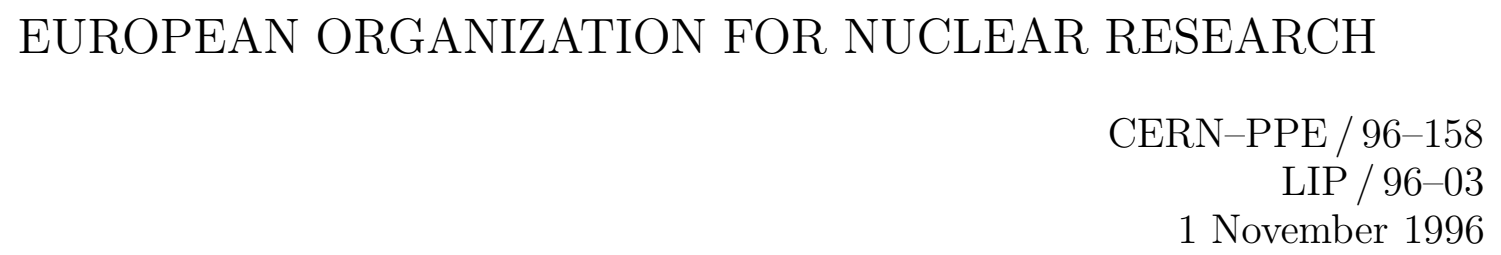

Hard probes in nucleus-nucleus collisions

\author{
C. Lourenço \\ CERN PPE, CH-1211 Geneva 23, Switzerland
}

\begin{abstract}
The present knowledge on hard processes in the context of heavy ion collisions is reviewed, with particular emphasis on $\mathrm{J} / \psi$ production. The $\mathrm{p}$-A data on charmonia production from Fermilab experiments is shown to be in excelent agreement with the p-A data collected at CERN. The simultaneous analysis of all existing p-A data reaches a precision which allows us to rule out some preconceived ideas, setting a good frame against which the data collected with ion beams at CERN can be compared.
\end{abstract}

Summary talk presented at the 12th International Conference on Ultra-Relativistic Nucleus-Nucleus Collisions (Quark Matter '96), Heidelberg, Germany, May 1996. 


\title{
Hard probes in nucleus-nucleus collisions
}

\author{
Carlos Lourenço
}

CERN PPE, CH-1211 Geneva 23, Switzerland

The present knowledge on hard processes in the context of heavy ion collisions is reviewed, with particular emphasis on $\mathrm{J} / \psi$ production. The $\mathrm{p}-\mathrm{A}$ data on charmonia production from Fermilab experiments is shown to be in excelent agreement with the p-A data collected at CERN. The simultaneous analysis of all existing p-A data reaches a precision which allows us to rule out some preconceived ideas, setting a good frame against which the data collected with ion beams at CERN can be compared.

\section{Introduction}

The first data on $\mathrm{J} / \psi$ production in nucleus-nucleus collisions were collected 10 years ago, at the CERN SPS, by the NA38 experiment, with the $200 \mathrm{GeV}$ per nucleon oxygen beam.

Although the experiment had been planned to search for thermal dimuons, a paper by Matsui and Satz [1] redirected attention by concluding that "there appears to be no mechanism for $\mathrm{J} / \psi$ suppression in nuclear collisions except the formation of a deconfining plasma, and if such a plasma is produced, there seems to be no way to avoid $\mathrm{J} / \psi$ suppression."

In fact, already at QM '87, the NA38 collaboration presented evidence of a substantial decrease of the ratio $\psi /$ Continuum, both from $\mathrm{p}-\mathrm{U}$ to $\mathrm{O}-\mathrm{U}$ collisions and versus $E_{\mathrm{T}}$ within $\mathrm{O}-\mathrm{U}$ [2]. These observations were soon confirmed with $\mathrm{S}-\mathrm{U}$ data [3].

Although the exciting interpretation of this "J/ $/ \psi$ suppression" as a signal of QGP formation was always present, some alternative explanations of a more "classical" nature were soon proposed.

Several developments helped to clarify the interpretation of the data:

- Concerning the absolute cross sections, Fermilab experiment E772 observed that $\mathrm{J} / \psi$ production is, in $\mathrm{p}-\mathrm{A}$ collisions, proportional to $\mathrm{A}^{\alpha}$, with an $\alpha$ value smaller than the one previously reported by NA3 (0.94 \pm 0.03 [四]) and used in early NA38 papers [5].

- Several measurements of $B_{\mu \mu} \sigma^{\psi}$ in p-A collisions were obtained by NA38, leading to a much more complete and accurate reference in the study of the ion data.

- Higher statistics in the S-U data set allowed to use the high mass Drell-Yan as the reference in the $E_{\mathrm{T}}$ dependent (relative) studies, replacing $\psi /$ Cont by $\psi / \mathrm{DY}$. Furthermore, the isospin correction $(\sim 10 \%)$ required when comparing the proton 
to the (isoscalar) sulphur induced collisions lead to a smaller "suppression" relative to what had been reported before, using $\psi /$ Cont.

At the same time, information on the $\psi$ ' became available, revealing a "suppression" much beyond the nuclear absorption expected from the $\mathrm{p}-\mathrm{A}$ data.

- The absolute cross sections of direct $\mathrm{J} / \psi$ and $\psi \prime$ production were found to be far too high relative to the values calculated with the colour singlet model. Although this was known to be the case already from fixed target data [6], the new (high $p_{\mathrm{T}}$ ) data from CDF [7], clearly identifying the fraction of $\mathrm{J} / \psi$ coming from beauty and $\chi_{c}$ decays, triggered a major change in the theoretical understanding of charmonia production [8]. Contrary to the paradigm of the colour singlet model, a substantial fraction of the $\mathrm{J} / \psi$ and $\psi /$ cross section is now believed to come from binding of $c \bar{c}$ pairs produced in a colour octet state.

Taking into account all these developments, some of them only available after QM '95, Kharzeev and Satz [9] were able to explain the phenomenological fit made earlier by Gerschel and Hüfner [10], by associating the absorption cross section of around 6-7 mb to the $c \bar{c} g$ state crossing the nuclear matter, rather than to a fully formed $\mathrm{J} / \psi$, for which they had previously calculated a much smaller cross section [11].

The further suppression of the $\psi \prime$ observed in S-U collisions is explained [9] by the formation of a (confined) hadronic medium in the later stages of the collision. These "comovers" can easily break the loosely bound, and by then fully formed, $\psi /$ while leaving the $\mathrm{J} / \psi$ unchanged, since confined gluons are too soft to overcome the $\mathrm{J} / \psi$ 's $(\sim 640 \mathrm{MeV})$ binding energy.

This was the status of the charmonia front just before the results from the $\mathrm{Pb}$ data collected in December 1995 became available. These new results [12 certainly open another very exciting chapter in the $\mathrm{J} / \psi$ saga, and the people attending QM '96 will remember the lively discussions held inside and outside of the conference room.

Some of the proposed "explanations" are not accurate enough to be seriously considered. For instance, it is not reasonable to reproduce the $\mathrm{Pb}-\mathrm{Pb}$ data at the price of missing the p-A and S-U points. Also, we cannot mix the $\psi /$ Cont values of S-U (presented for the last time at $\mathrm{QM}$ '91) with the $\psi / \mathrm{DY}$ values of $\mathrm{Pb}-\mathrm{Pb}$, the latter variable certainly being much better (remember the enhancement observed in the intermediate mass region [13], which might affect the "continuum").

The precision and diversity of the measurements now available certainly deserves equally accurate theoretical descriptions. The field of $\mathrm{J} / \psi$ production in heavy ion collisions has reached maturity and QM '96 should be considered as a transition point: the time has come to build realistic theoretical models that reproduce the data with an accuracy at the few percent level.

In this sense, it would be very helpful to develop a microscopic model (like RQMD, VENUS or HIJING) that would generate hard processes in a nuclear environment, including nuclear geometry effects, modifications of the parton distribution functions, etc. Of course, it would have to work also at SPS energies, not only for RHIC and LHC.

In the remaining part of this paper, the data presently available is presented in detail, both the directly measured values and, where they are required, those obtained after 


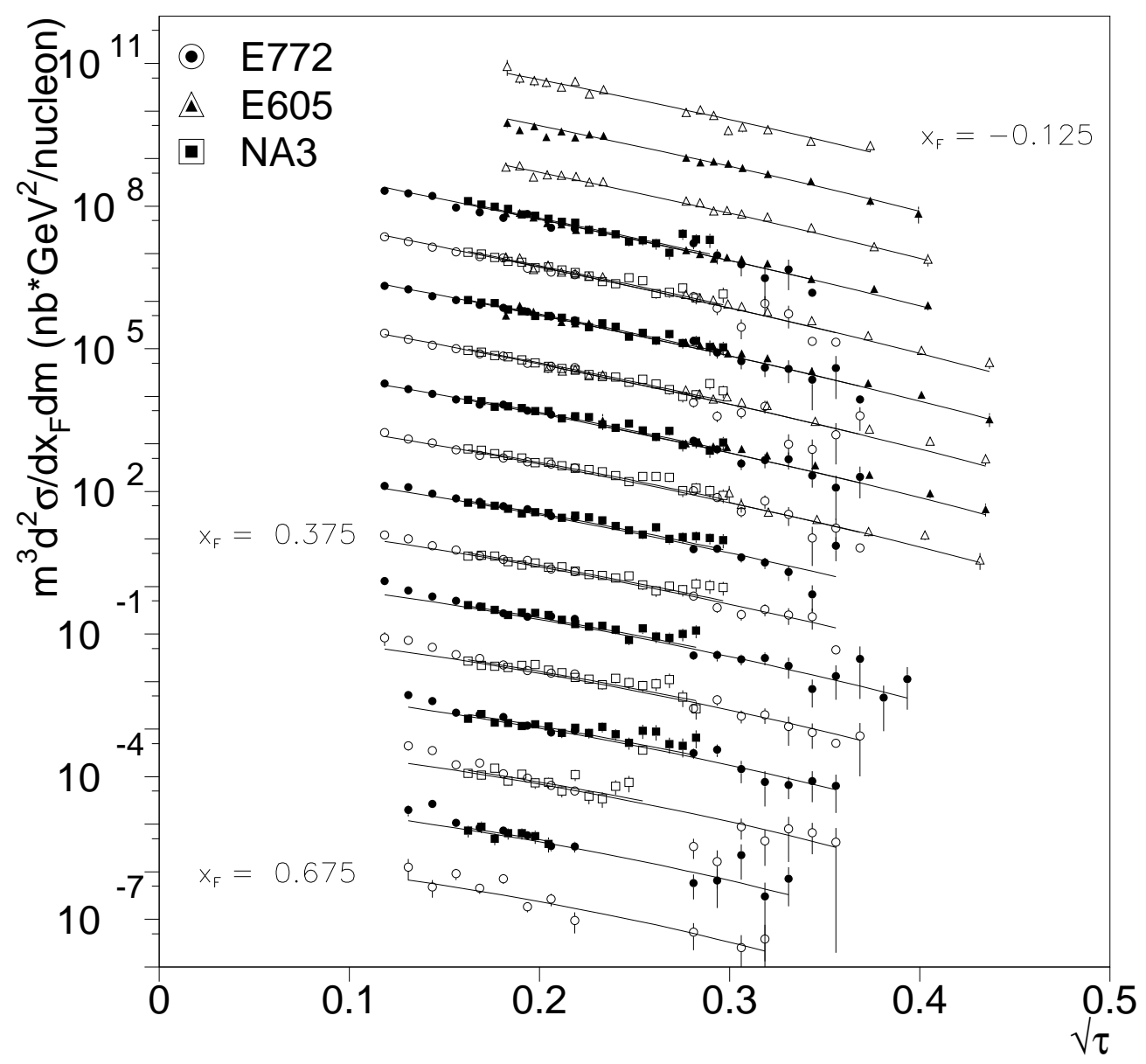

Figure 1. p-A Drell-Yan double differential cross section, per target nucleon. See text for details.

correcting for $\sqrt{s}$ dependences and phase space windows. Special emphasis is given to the p-A data, collected at FNAL and at CERN, enough by it self to rule out some ideas.

\section{Hard processes in p-A collisions}

\subsection{Drell-Yan production}

Figure 11 presents the double differential Drell-Yan cross section, $m^{3} \mathrm{~d}^{2} \sigma / \mathrm{d} m \mathrm{~d} x_{F}$, in $\mathrm{nb} \times \mathrm{GeV}^{2} /$ nucleon, versus the scaling variable $\sqrt{\tau}=m / \sqrt{s}$ (using mass bins of $0.5 \mathrm{GeV}$ ), for different $x_{\mathrm{F}}$ bins $(0.05$ wide). For presentation purposes, the points above (below) the $x_{F}=0.375$ data set have been successively multiplied (divided) by factors of 10 . This figure merges Drell-Yan data from three p-A dimuon experiments: E772, E605 and NA3 [14 [16]. The data are available as tables from the HEPDATA data base [17]. The beam energy was $800 \mathrm{GeV}$ in the Fermilab experiments and $400 \mathrm{GeV}$ in the case of NA3. Different targets were used: hydrogen (E772), copper (E605) and platinum (NA3). The 
agreement between the three experiments is excelent. The curves on the figure are the result of a leading order calculation using the CTEQ2M set of (NLL) quark distribution functions [18, multiplied by an appropriate $\mathrm{K}$ factor (around 1.5). The $\mathrm{K}$ factor is considerably reduced when the calculations are performed in NLO and becomes $\sim 1$ at NNLO [19].

The influence of the nuclear target, usually referred to as "the A-dependence", is quite simple in the case of Drell-Yan production. The cross section just scales linearly with the number of target nucleons, $\sigma_{p A}^{D Y}=\sigma_{0}^{D Y} \times A$. A closer look reveals that we have to count the number of protons and neutrons separately, since the Drell-Yan cross section depends on the charge of the annihilating quarks. In fact, the precision reached in DrellYan measurements is such that it has revealed differences between the structure functions of the $\bar{u}$ and $\bar{d}$ sea in the nucleons [20], a probable explanation for the violation of the Gottfried sum rule recently observed in deep-inelastic lepton scattering [21].
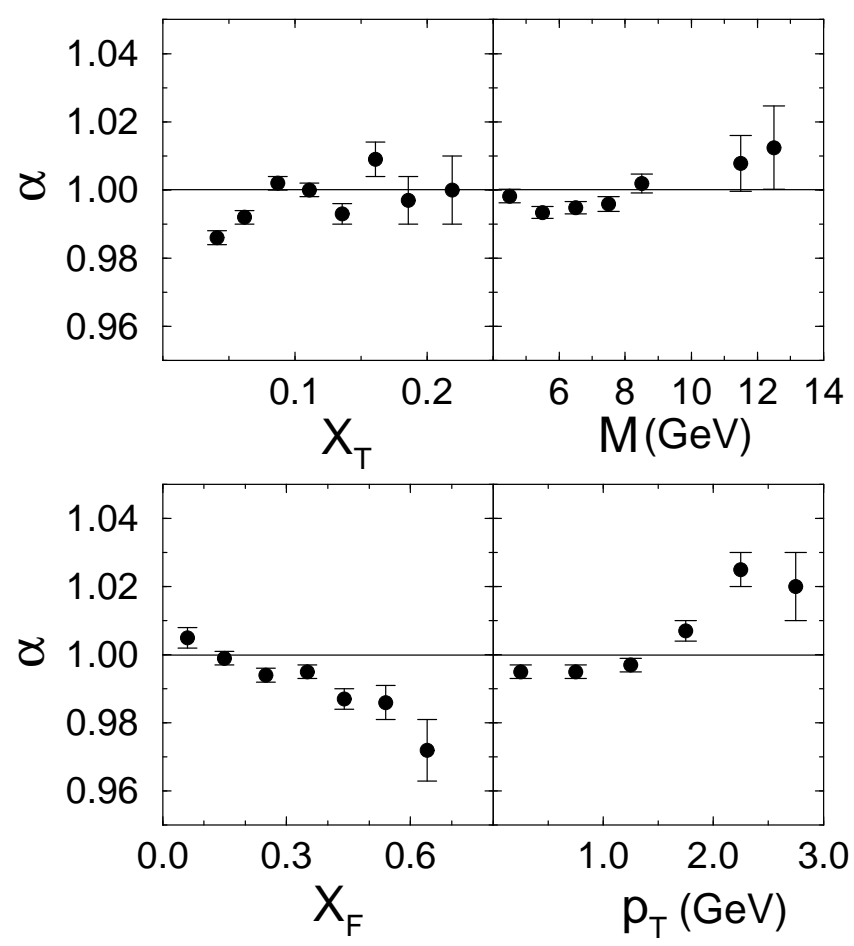

Figure 2. Drell-Yan A-dependence expressed as $\alpha$ versus $x_{\mathrm{T}}, \mathrm{m}, x_{\mathrm{F}}$ and $p_{\mathrm{T}}$. Notice the fine scale used in the vertical axis.

The dependence of Drell-Yan on A, for p-A collisions, can be expressed in terms of the $\alpha$ parameter, as done in figure 2, or by the ratios between the dimuon yields per nucleon in heavy targets relative to a light target, say deuterium, as presented in table 1 . 
Table 1

Ratios of DY, $\mathrm{J} / \psi$ and $\psi \prime$ cross sections between $\mathrm{p}-\mathrm{A}$ and $\mathrm{p}^{2} \mathrm{H}$, from E772 data.

\begin{tabular}{lccc}
\hline Target $(\mathrm{A})$ & $\mathrm{DY}$ & $\mathrm{J} / \psi$ & $\psi \prime$ \\
\hline $\mathrm{C}(12)$ & $1.003 \pm 0.0085$ & $0.851 \pm 0.0125$ & $0.855 \pm 0.0292$ \\
$\mathrm{Ca}(40)$ & $0.995 \pm 0.0061$ & $0.806 \pm 0.0087$ & $0.750 \pm 0.0271$ \\
$\mathrm{Fe} \mathrm{(56)}$ & $0.990 \pm 0.0065$ & $0.756 \pm 0.0102$ & $0.722 \pm 0.0357$ \\
$\mathrm{~W} \mathrm{(184)}$ & $0.986 \pm 0.0083$ & $0.619 \pm 0.0133$ & $0.623 \pm 0.0331$ \\
\hline
\end{tabular}

\section{2. $\mathbf{J} / \psi$ production}

Table 1 also includes the values measured by the E772 experiment [22] for the $\mathrm{J} / \psi$ and $\psi \prime$ resonances, first shown at the QM '90 conference.

Before comparing the results of the CERN experiments, NA38 and NA51, with the E772 data, in what concerns p-A collisions, we should notice that the $\alpha$ of $\mathrm{J} / \psi$ depends on $x_{\mathrm{F}}$, as is shown in figure 3 [22]. The E772 data on $\alpha^{\psi}$ is also presented in table 2 .

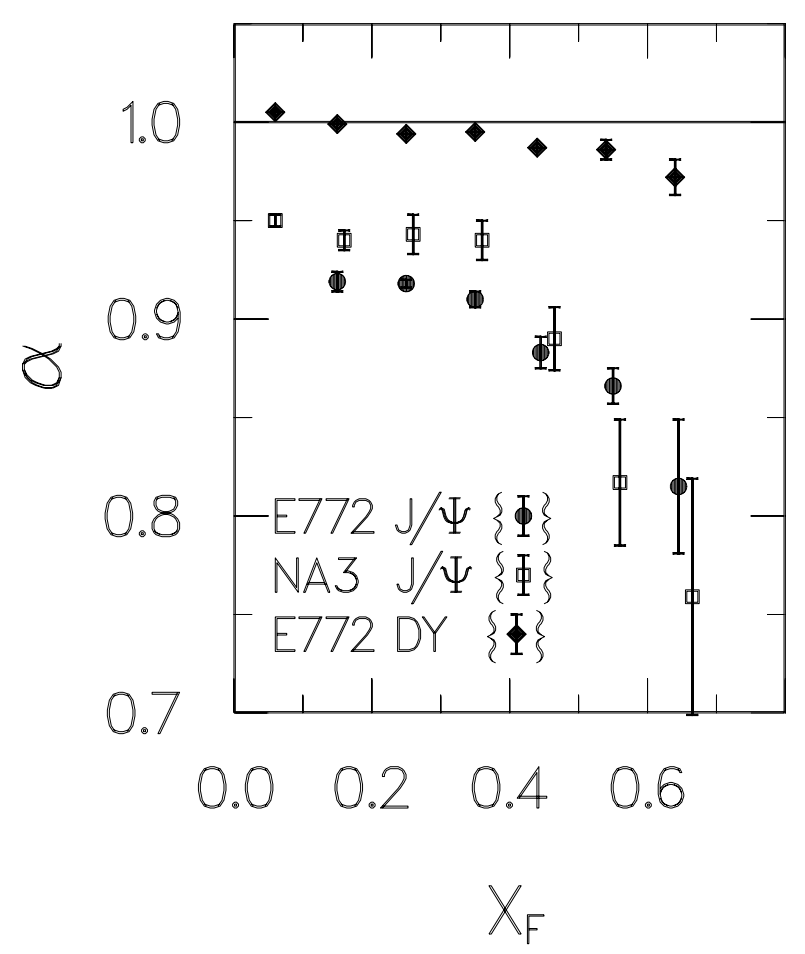

Figure 3. $x_{\mathrm{F}}$ dependence of $\alpha^{D Y}$ and $\alpha^{\psi}$.

Since the $\mathrm{J} / \psi$ cross section becomes negligible at high $x_{\mathrm{F}}$, the average value of $\alpha^{\psi}$, for positive $x_{\mathrm{F}}$, is around 0.91. In fact, the value $\alpha^{\psi}=0.920 \pm 0.008$ was published by E772 in Ref. 22 but the E789 collaboration uses the value $\alpha^{\psi}=0.90 \pm 0.02$ in Ref. 23.

We move now to the p-A measurements done at the CERN/SPS by the NA38 and NA51 
Table 2

Values of $\alpha^{\psi}$ versus $x_{\mathrm{F}}$, from E772 data.

\begin{tabular}{cc}
\hline$x_{\mathrm{F}}$ & $\alpha^{\psi}$ \\
\hline 0.15 & $0.919 \pm 0.005$ \\
0.25 & $0.918 \pm 0.002$ \\
0.35 & $0.910 \pm 0.004$ \\
0.45 & $0.883 \pm 0.008$ \\
0.55 & $0.866 \pm 0.009$ \\
0.65 & $0.815 \pm 0.034$ \\
\hline
\end{tabular}

collaborations. These data are certainly the best to study the (target) nuclear effects on $\mathrm{J} / \psi$ production as a reference for the study of the heavy ion data, since they were collected with the same dimuon spectrometer, therefore being in the same kinematical window. However, we should start by checking that there is compatibility between the p-A results of NA38/51 and the observations done at Fermilab.

The p-A results of NA38 and NA51, in what concerns $\mathrm{J} / \psi$ cross sections, are presented in table 3. These values correspond to the phase space domain $(\Delta)$ covered by the detector, $0<y^{*}<1$ and $\left|\cos \theta_{C S}\right|<0.5, y^{*}$ and $\theta_{C S}$ being the center of mass rapidity and the dimuon decay angle in the Collins-Sopper frame.

Table 3

Cross sections for $\mathrm{J} / \psi$ production in $\mathrm{p}$ - A collisions, times b.r. into muons, as measured by the NA38 and NA51 collaborations. See the text for details.

\begin{tabular}{lccc}
\hline & $\begin{array}{c}p_{\text {beam }} \\
(\mathrm{GeV})\end{array}$ & $\begin{array}{c}B \sigma_{\Delta}^{\psi} \\
(\text { nb/nucleus })\end{array}$ & $\begin{array}{c}B \sigma_{\Delta}^{\psi} / \mathrm{A} \\
(\mathrm{nb} / \text { nucleon })\end{array}$ \\
\hline $\mathrm{pp}$ & 450 & $4.41 \pm 0.52$ & $4.41 \pm 0.52$ \\
$\mathrm{p}-{ }^{2} \mathrm{H}$ & 450 & $9.16 \pm 1.05$ & $4.58 \pm 0.52$ \\
\hline $\mathrm{p}-\mathrm{C}$ & 450 & $46.7 \pm 5.3$ & $3.89 \pm 0.44$ \\
$\mathrm{p}-\mathrm{Al}$ & 450 & $93.8 \pm 10.7$ & $3.47 \pm 0.40$ \\
$\mathrm{p}-\mathrm{Cu}$ & 450 & $216 \pm 24$ & $3.43 \pm 0.38$ \\
$\mathrm{p}-\mathrm{Cu}$ & 200 & $104 \pm 23$ & $1.65 \pm 0.37$ \\
$\mathrm{p}-\mathrm{W}$ & 450 & $566 \pm 65$ & $3.08 \pm 0.36$ \\
$\mathrm{p}-\mathrm{W}$ & 200 & $263 \pm 18$ & $1.43 \pm 0.10$ \\
$\mathrm{p}-\mathrm{U}$ & 200 & $326 \pm 69$ & $1.37 \pm 0.29$ \\
\hline
\end{tabular}

In order to fit the $\mathrm{J} / \psi$ cross sections measured in $\mathrm{p}-\mathrm{A}$ collisions by the NA38 and NA51 collaborations to the $\mathrm{A}^{\alpha}$ form, we must first correct for the different beam energies used. Indeed, the data was collected either with $200 \mathrm{GeV}$ or $450 \mathrm{GeV}$ proton beams. Since the heavy ion data was collected at $200 \mathrm{GeV} /$ nucleon $(158 \mathrm{GeV}$ in the case of the Pb beam) we take $\sqrt{s}=19.4 \mathrm{GeV}$ as the reference and rescale the other values.

The $\sqrt{s}$ dependence of the $\mathrm{J} / \psi$ cross section (times b.r. into muons) is displayed in figure 4 , basically taken from fig. 11 of Ref. 23 but with a few more points. To make this 
figure, the "per nucleon" cross sections were evaluated using an $\alpha$ of 0.91 (it would look essentially the same with 0.92).

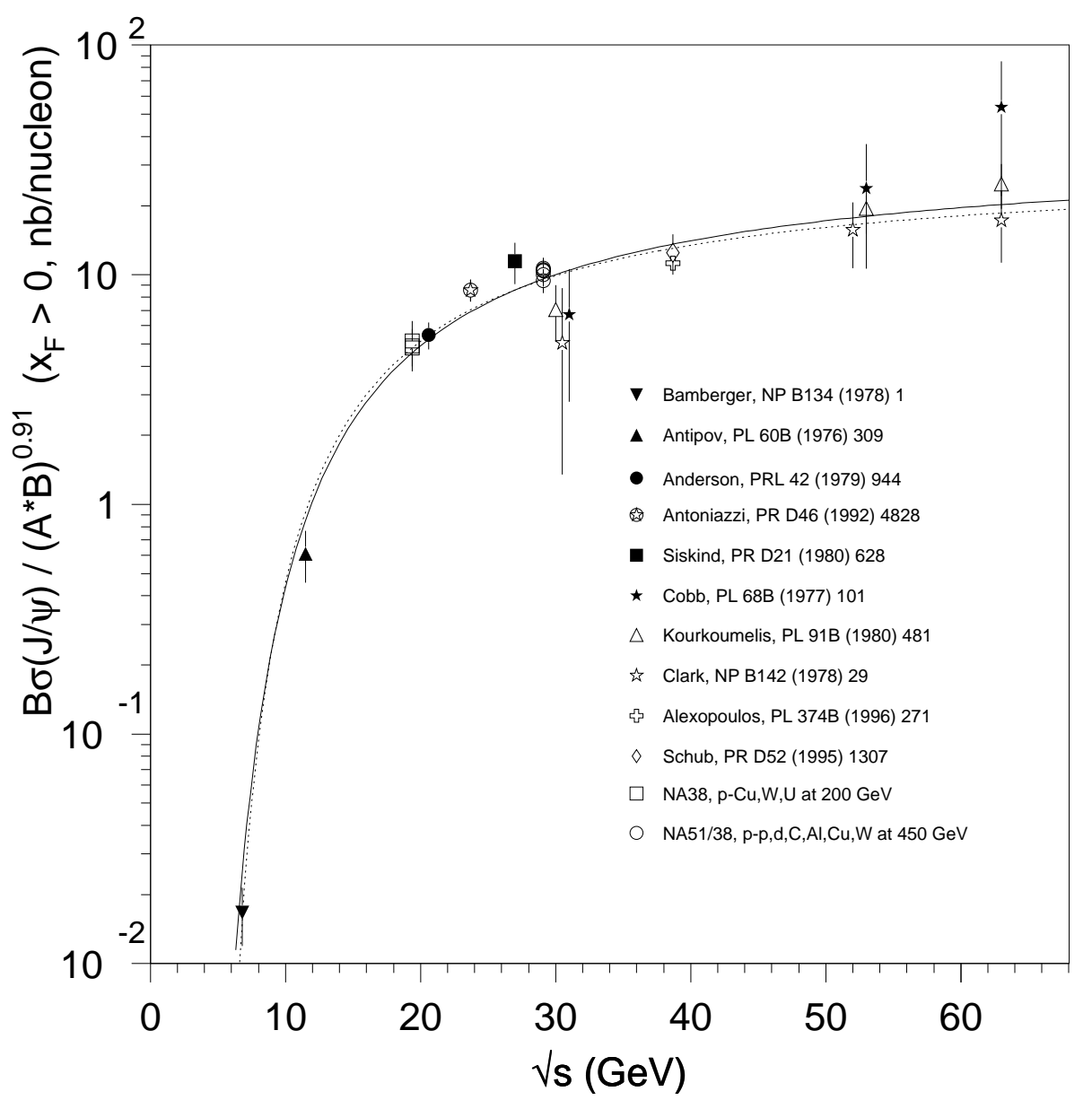

Figure 4. Energy dependence of the $\mathrm{J} / \psi$ cross section.

Notice that we have included in this figure the values measured by NA51 and NA38 (after converting them to the positive $x_{\mathrm{F}}$ domain, see below). They are in excelent agreement among them selfs and fit very well within the other measurements.

The solid line on the figure (the dotted line will be explained later) is

$\frac{B \sigma^{\psi}}{A^{0.91}}(\sqrt{s})=37 \times\left(1-\frac{3.097}{\sqrt{s}}\right)^{12} \quad$, in nb/nucleon

We have used this function to rescale the $450 \mathrm{GeV}$ values, the result being given in table 4 . In the last column of the same table are the corresponding values in the $x_{\mathrm{F}}>0$ phase space domain. We have multiplied the measured values by a factor of 2 to account 
Table 4

Cross sections for $\mathrm{J} / \psi$ production in $\mathrm{p}-\mathrm{A}$ collisions, times b.r. into muons, after corrections. See the text for details.

\begin{tabular}{lccc}
\hline & $\begin{array}{c}p_{\text {beam }} \\
(\mathrm{GeV})\end{array}$ & $\begin{array}{c}B \sigma_{\Delta}^{\psi} / \mathrm{A} \text { (rescaled) } \\
(\mathrm{nb} / \text { nucleon })\end{array}$ & $\begin{array}{c}B \sigma_{x_{\mathrm{F}}>0}^{\psi} / \mathrm{A} \text { (rescaled) } \\
(\mathrm{nb} / \text { nucleon })\end{array}$ \\
\hline $\mathrm{pp}$ & 450 & $2.11 \pm 0.25$ & $4.52 \pm 0.54$ \\
$\mathrm{p}-{ }^{2} \mathrm{H}$ & 450 & $2.19 \pm 0.25$ & $4.69 \pm 0.54$ \\
\hline $\mathrm{p}-\mathrm{C}$ & 450 & $1.86 \pm 0.21$ & $3.98 \pm 0.45$ \\
$\mathrm{p}-\mathrm{Al}$ & 450 & $1.66 \pm 0.19$ & $3.55 \pm 0.41$ \\
$\mathrm{p}-\mathrm{Cu}$ & 450 & $1.64 \pm 0.18$ & $3.51 \pm 0.39$ \\
$\mathrm{p}-\mathrm{Cu}$ & 200 & $1.65 \pm 0.37$ & $3.53 \pm 0.79$ \\
$\mathrm{p}-\mathrm{W}$ & 450 & $1.47 \pm 0.17$ & $3.15 \pm 0.36$ \\
$\mathrm{p}-\mathrm{W}$ & 200 & $1.43 \pm 0.10$ & $3.06 \pm 0.21$ \\
$\mathrm{p}-\mathrm{U}$ & 200 & $1.37 \pm 0.29$ & $2.93 \pm 0.62$ \\
\hline
\end{tabular}

for the $\cos \theta_{C S}$ coverage (assuming a uniform decay angle distribution) and by a factor 1.07 to convert the $y *$ range into the positive $x_{\mathrm{F}}$ window.

From the rescaled values presented in table 4 , we can fit an $\mathrm{A}^{\alpha}$ function and extract $\alpha^{\psi}$. The result is $0.908 \pm 0.029$, excluding the pp point (otherwise it is $0.919 \pm 0.021$ ).

This is in excelent agreement with the results from Fermilab E772/E789 experiments.

\section{3. $\psi$ ' production}

$\psi \prime$ production in $\mathrm{p}$ - A collisions is easier to study through the ratio $\psi^{\prime} / \psi$, since the $\mathrm{J} / \psi$ has well understood energy and A dependencies. The values of the ratio $\psi^{\prime} / \psi$, ratio of production cross-sections times branching ratios into muon pairs, measured in $\mathrm{p}-\mathrm{A}$ collisions 24, 25], are collected in table 5.

Table 5

Values of the $\psi^{\prime} / \psi$ ratio obtained in p-A collisions.

\begin{tabular}{lccc}
\hline & $p_{\text {beam }}(\mathrm{GeV})$ & $B \sigma^{\psi^{\prime}} / B \sigma^{\psi}(\%)$ & Exp.t \\
\hline $\mathrm{p}-\mathrm{H}_{2}$ & 450 & $1.69 \pm 0.03$ & NA51 \\
$\mathrm{p}-\mathrm{D}_{2}$ & 450 & $1.80 \pm 0.03$ & NA51 \\
$\mathrm{p}-\mathrm{C}$ & 450 & $1.90 \pm 0.13$ & NA38 \\
$\mathrm{p}-\mathrm{Al}$ & 450 & $1.36 \pm 0.35$ & NA38 \\
$\mathrm{p}-\mathrm{Cu}$ & 450 & $1.68 \pm 0.11$ & NA38 \\
$\mathrm{p}-\mathrm{W}$ & 450 & $1.59 \pm 0.13$ & NA38 \\
$\mathrm{p}-\mathrm{W}$ & 200 & $1.80 \pm 0.17$ & NA38 \\
$\mathrm{p}-\mathrm{U}$ & 200 & $1.77 \pm 0.22$ & NA38 \\
\hline $\mathrm{p}-\mathrm{p}$ & $\sqrt{s}=63$ & $1.9 \pm 0.6$ & ISR \\
$\mathrm{p}-\mathrm{Li}$ & 300 & $1.88 \pm 0.26 \pm 0.05$ & E 705 \\
$\mathrm{p}-\mathrm{Be}$ & 400 & $1.7 \pm 0.5$ & E 288 \\
$\mathrm{p}-\mathrm{Si}$ & 800 & $1.65 \pm 0.20$ & E 771 \\
$\mathrm{p}-\mathrm{Au}$ & 800 & $1.80 \pm 0.1 \pm 0.2$ & E 789 \\
\hline
\end{tabular}


These values show that the ratio between $\psi^{\prime}$ and $\mathrm{J} / \psi$ production cross sections is independent of the target nuclei and (at high energies) of $\sqrt{s}$. In fact, we can even add values obtained with other beam particles [24, 26] $\left(\pi^{+}, \pi^{-}, \overline{\mathrm{p}}, \gamma\right)$ without changing the picture.

This observation is a clear indication that these charmonia bound states are actually only formed as such outside the target nucleus, at least when they are fast enough (at positive $x_{F}$ ). It would certainly be very interesting to see what happens when the $c \bar{c}$ state is slow enough to become a $\mathrm{J} / \psi$ or $\psi \prime$ within nuclear matter [27]. Present (p-A) dimuon experiments are unable to detect the correspondingly slow decay muons but this might soon be overcome by an "inverse kinematics experiment", sending the SPS Pb beam on a light target.

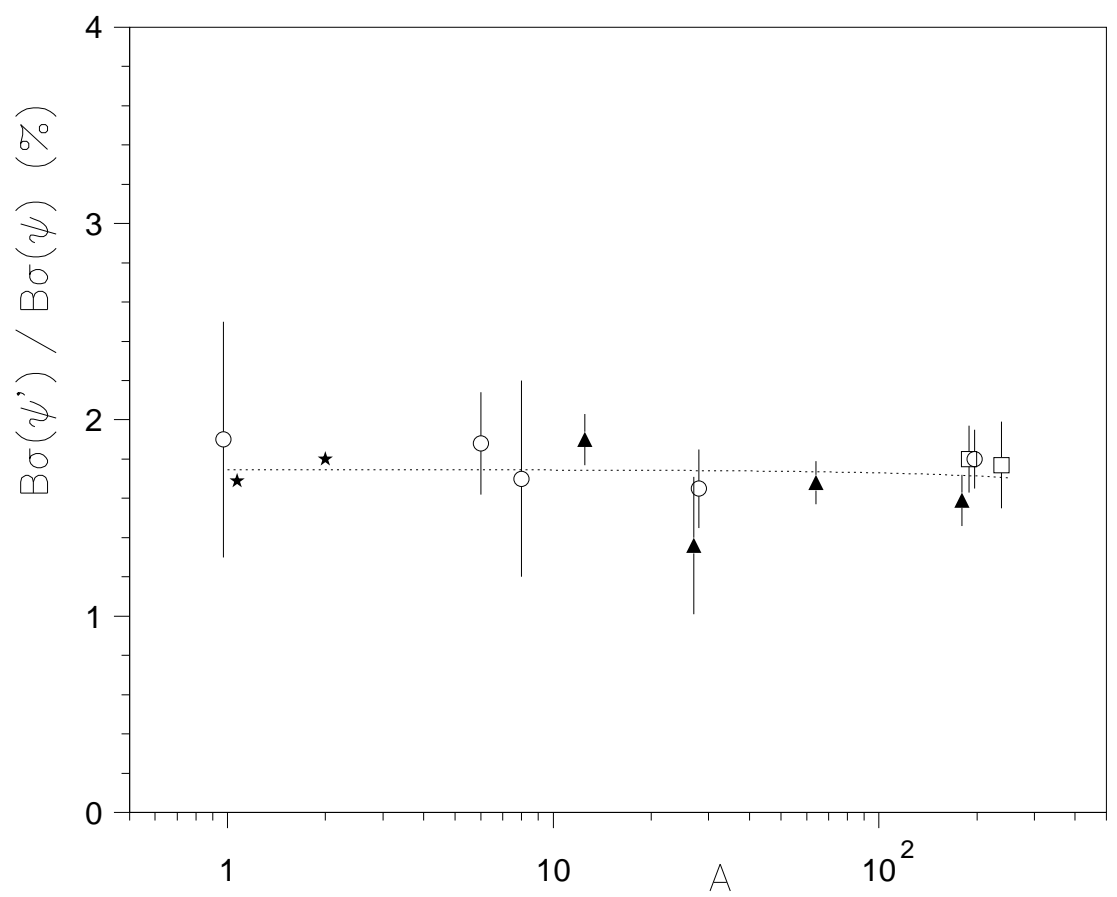

Figure 5. Target dependence of the $\psi^{\prime} / \psi$ ratio, in p-A collisions.

The data of table 5 have been plotted versus $\mathrm{A}$ in figure 5 and versus $\sqrt{s}$ in figure 6 . In these figures, some of the points were slightly displaced in the horizontal axis (otherwise a few points would be indistinguishable).

The dotted curves in these plots are the result from linear fits to the data, the slopes being compatible with zero for both the A and the $\sqrt{s}$ cases, $(-1.6 \pm 4.2) \times 10^{-4}$ and $(-8.1 \pm 79) \times 10^{-4}$, respectively.

Even if $\alpha^{\psi^{\prime}}$ would be smaller than $\alpha^{\psi}$ by only 0.01 , that would imply a decrease of $\psi^{\prime} / \psi$ from pp to $\mathrm{p}-\mathrm{U}$ of $5.6 \%$, already quite difficult to accomodate by these measurements. Therefore, models which predict a substantially different A dependence for the $\mathrm{J} / \psi$ and 


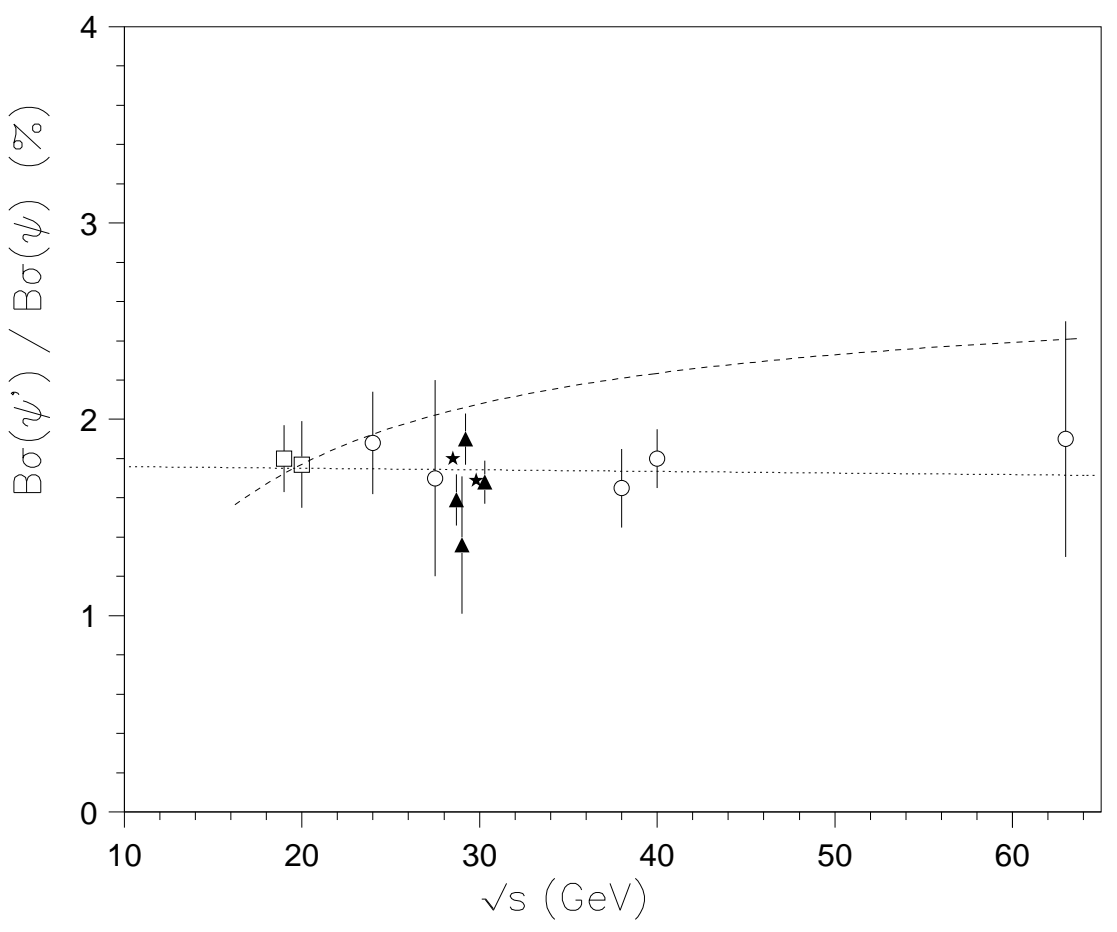

Figure 6. Energy dependence of the $\psi^{\prime} / \psi$ ratio, in p-A collisions.

$\psi /$ resonances, in $\mathrm{p}-\mathrm{A}$ collisions, are in clear contradiction with the data.

Figure 6 also contains a second (dashed) curve, which represents the evolution with $\sqrt{s}$ of the $\psi^{\prime} / \psi$ ratio expected if we assume the $\psi$ ' cross section to depend on $\sqrt{s}$ according to equation 1, with the parameter $3.097 \mathrm{GeV}$ replaced by $3.685 \mathrm{GeV}$. The fact that this curve (normalised in the plot at $\sqrt{s}=19.4 \mathrm{GeV}$ ) completely fails to go through the data points, shows very well how incorrect such an assumption is, and reveals that this parameter should not be directly associated to the mass of the particle under study. In fact, the best fit to the points in figure 1 is provided by $31.5 \times(1-4.4 / \sqrt{s})^{7.3}$ (dotted line in that figure).

\section{Hard processes in nucleus-nucleus collisions}

Drell-Yan differential cross sections (corrected for detector specific acceptance and smearing effects) are not yet available for nucleus-nucleus collisions. Work in this direction is still in progress within the NA38/50 collaborations. Therefore, it is not yet possible to compare the data with NLO calculations (not available in the form of event generators). Although the nuclear effects on the parton distribution functions are expected to be small, in the kinematical window of NA38/50, they are certainly non-zero and it would be very important to actually measure them rather than just neglect their existence. For the moment, the fact that the same $\mathrm{K}$ factor (relative to a certain LO calculation) is required by the $\mathrm{p}-\mathrm{A}$ and ion data [12] is the best argument to say that Drell-Yan is a good reference in the studies of charmonia production by ion collisions. 
The $\mathrm{J} / \psi$ cross sections measured in nucleus-nucleus collisions, by the NA38 and NA50 collaborations, are collected in table 6 , before and after $\sqrt{s}$ and phase space corrections.

Table 6

$\mathrm{J} / \psi$ cross sections, times b.r. into muons, measured by the NA38 and NA50 collaborations in B-A collisions.

\begin{tabular}{lcccc}
\hline & $\begin{array}{c}p_{\text {beam }} \\
(\mathrm{GeV})\end{array}$ & $\begin{array}{c}B \sigma_{\Delta}^{\psi} \\
(\mu \mathrm{b})\end{array}$ & $\begin{array}{c}B \sigma_{\Delta}^{\psi} /(\mathrm{B} \times \mathrm{A})(\text { rescaled }) \\
(\mathrm{nb} / \text { nucleon })\end{array}$ & $\begin{array}{c}B \sigma_{x_{\mathrm{F}}>0}^{\psi} /(\mathrm{B} \times \mathrm{A}) \text { (rescaled) } \\
(\mathrm{nb} / \text { nucleon })\end{array}$ \\
\hline $\mathrm{O}-\mathrm{Cu}$ & 200 & $1.26 \pm 0.13$ & $1.25 \pm 0.13$ & $2.68 \pm 0.28$ \\
$\mathrm{O}-\mathrm{U}$ & 200 & $4.42 \pm 0.46$ & $1.16 \pm 0.12$ & $2.48 \pm 0.26$ \\
$\mathrm{~S}-\mathrm{U}$ & 200 & $7.69 \pm 0.76$ & $1.01 \pm 0.10$ & $2.16 \pm 0.21$ \\
\hline $\mathrm{Pb}-\mathrm{Pb}$ & 158 & $19.0 \pm 1.4$ & $0.59 \pm 0.04$ & $1.26 \pm 0.09$ \\
\hline
\end{tabular}

The light ion collisions are in excelent agreement with the extrapolation of the proton data "nuclear absorption" curve. Indeed, if we include the oxygen and sulphur points in the fit to a $(\mathrm{B} \times \mathrm{A})^{\alpha}$ function, the value of $\alpha$ stays the same $(0.911 \pm 0.016)$ as before. This smooth transition from $\mathrm{p}-\mathrm{A}$ to $\mathrm{O}, \mathrm{S}-\mathrm{A}$ collisions is illustrated in figure $\mathrm{O}$.

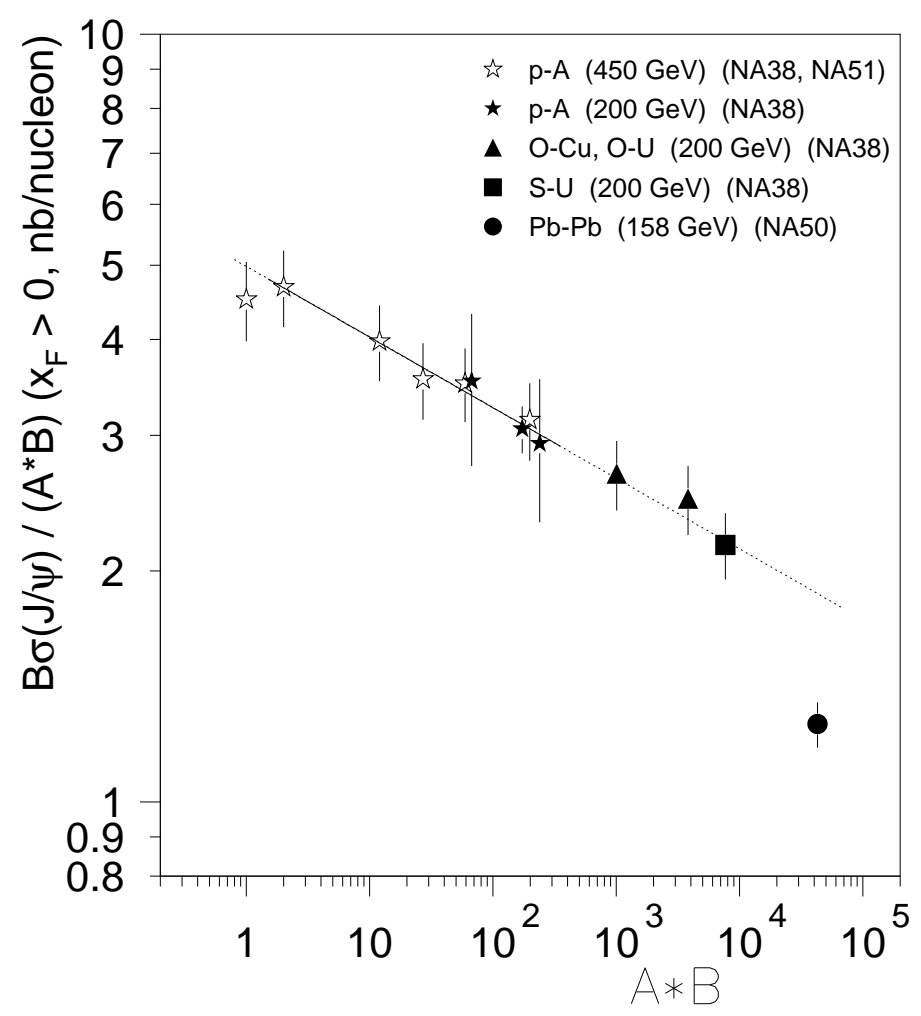

Figure 7. $\mathrm{J} / \psi$ production cross sections (times b.r.) versus $\mathrm{A} \times \mathrm{B}$. The straight line, in this $\log -\log$ plot, corresponds to $\alpha=0.908$, the dotted part being an extrapolation from the fitted region. 
Contrary to what happens in the light ion region, the $\mathrm{Pb}-\mathrm{Pb}$ point is seen to be away from the "expected" production cross section, which is $50 \%$ higher than the measured value. This is a strong indication that something else besides "nuclear absorption" is happening to the $\mathrm{J} / \psi$ in $\mathrm{Pb}-\mathrm{Pb}$ collisions.

There are more precise ways of checking whether nuclear absorption alone can explain the observed evolution of $\mathrm{J} / \psi$ production yields. On the vertical axis we can use the ratio of $\mathrm{J} / \psi$ to Drell-Yan yields to reduce the systematic errors resulting from absolute normalisations. To explore the dependence of $\mathrm{J} / \psi$ production on the centrality of the ion collisions, we can subdivide the event samples according to some global variable, like $E_{\mathrm{T}}$ or $E_{\mathrm{ZDC}}$, sensitive to the geometry of the collision. In fact, the best variable within the "nuclear absorption" framework is the amount of nuclear matter crossed by the pre-J/ $\psi$ state on its way out, the famous L introduced by Gerschel and Hüfner [10.

Figure 8 attempts to merge in a single plot the variation with $\mathrm{L}$ of both the $\mathrm{J} / \psi$ and

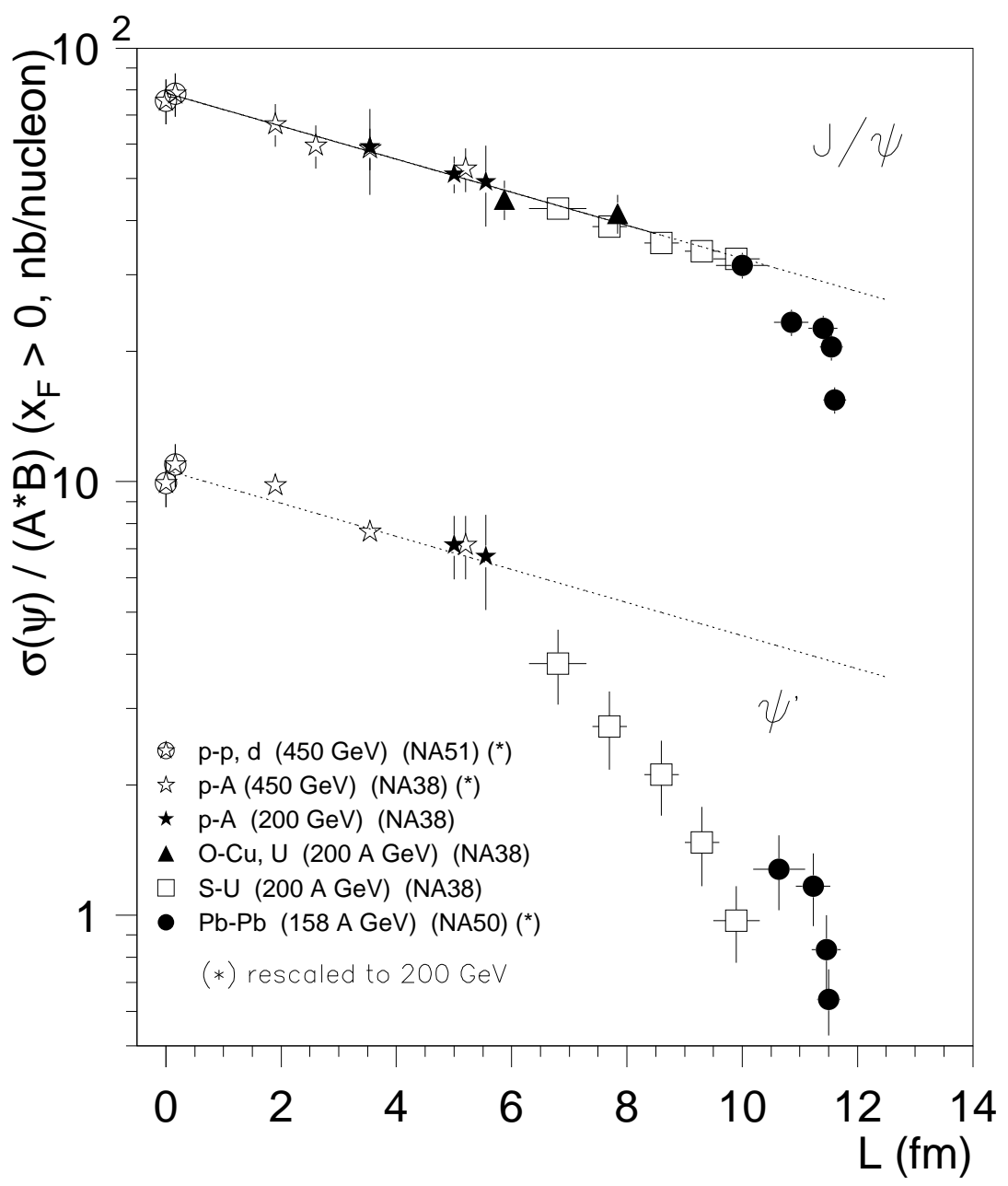

Figure $8 . \mathrm{J} / \psi$ and $\psi$ ' production cross sections versus L. The (parallel) lines correspond to $\exp (-\rho \sigma \mathrm{L})$, with $\rho \sigma=0.088 \mathrm{fm}^{-1}$. 
$\psi \prime$ cross sections. To cope with the range in the vertical scale, the branching ratios into muons were corrected for. The $(\mathrm{S}-\mathrm{U}$ and $\mathrm{Pb}-\mathrm{Pb}) \mathrm{L}$ dependent $\sigma^{\psi}$ values were obtained from $\psi / \mathrm{DY}$ using the all- $E_{\mathrm{T}}$ values for normalisation and assuming that DY does not depend on L (in other words, the integrated point is replaced by 5 points keeping the same average). The $\sigma^{\psi^{\prime}}$ points were obtained from the $\psi^{\prime} / \psi$ values (with some art to combine the different centrality bins used in the $\mathrm{J} / \psi$ and $\psi \prime$ analyses [12]).

We can see that the nuclear absorption model reproduces quite well all the $\mathrm{J} / \psi$ points up to the most peripheral $\mathrm{Pb}-\mathrm{Pb}$ collisions. The remaining $\mathrm{Pb}-\mathrm{Pb}$ points reveal the existence of a new "suppression" mechanism, L not being any longer an appropriate parameter.

The $\psi$ ' points reveal that after $\mathrm{L} \sim 6 \mathrm{fm}$ the two charmonia states are already fully formed and start feeling in a different way the medium they cross. The nuclear matter path length alone is no longer enough to correctly parametrise the $\psi$ ' behaviour, revealing that a dense hadronic medium is formed in $\mathrm{S}-\mathrm{U}$ collisions.

To understand whether the new $\mathrm{Pb}-\mathrm{Pb}$ data on charmonia production is more than just a "hint" of QGP formation, accurate studies will have to be pursued, much beyond the "L framework".

For instance, a correct understanding of the S-U data must consider that about $8 \%$ of the observed $\mathrm{J} / \psi$ yield actually come from $\psi$ ' decays. Since the $\psi \prime$ is significantly suppressed in S-U collisions, a certain fraction of the $\mathrm{J} / \psi$ yield must also be suppressed.

To conclude, table 7 collects useful information for further studies of $\mathrm{J} / \psi$ production from $\mathrm{p}-\mathrm{A}$ to $\mathrm{S}-\mathrm{U}$ and $\mathrm{Pb}-\mathrm{Pb}$ collisions, as a function of $E_{\mathrm{T}}$. Notice, however, that the $E_{\mathrm{T}}$ scales are not the same in the NA38 and NA50 experiments, due to the different rapidity windows covered by the calorimeters: $1.7<\eta<4.1$ in NA38 and $1.1<\eta<2.3$ in NA50. The resolution of this non-trivial problem would require a few more pages.

Table 7

$E_{\mathrm{T}}$ dependence of $\mathrm{J} / \psi$ yields: $\psi / \mathrm{DY}$ and after normalisation to the integrated cross section and to the pp value.

\begin{tabular}{ccccc}
\hline & $\begin{array}{c}E_{\mathrm{T}} \\
(\mathrm{GeV})\end{array}$ & $\psi / \mathrm{DY}$ & $\begin{array}{c}B \sigma_{\mathrm{BA}}^{\psi} /(\mathrm{B} \mathrm{A}) \\
(\mathrm{nb} / \text { nucleon })\end{array}$ & $\sigma_{\mathrm{BA}}^{\psi} /\left(\mathrm{B} \mathrm{A} \sigma_{\mathrm{pp}}^{\psi}\right)$ \\
\hline $\mathrm{S}-\mathrm{U}$ & $\mathrm{All}$ & $21.4 \pm 0.24$ & $1.01 \pm 0.10$ & $0.48 \pm 0.07$ \\
\hline 25 & $25.2 \pm 0.69$ & $1.19 \pm 0.12$ & $0.56 \pm 0.09$ \\
42 & $22.8 \pm 0.56$ & $1.08 \pm 0.11$ & $0.51 \pm 0.08$ \\
57 & $21.0 \pm 0.48$ & $0.99 \pm 0.10$ & $0.47 \pm 0.07$ \\
71 & $20.2 \pm 0.44$ & $0.95 \pm 0.10$ & $0.45 \pm 0.07$ \\
& 82 & $19.2 \pm 0.44$ & $0.91 \pm 0.09$ & $0.43 \pm 0.07$ \\
\hline $\mathrm{Pb}-\mathrm{Pb}$ & $\mathrm{All}$ & $11.9 \pm 0.4$ & $0.59 \pm 0.04$ & $0.28 \pm 0.04$ \\
\hline & $17.8 \pm 2.20$ & $0.88 \pm 0.13$ & $0.42 \pm 0.08$ \\
& 59 & $13.2 \pm 0.99$ & $0.65 \pm 0.07$ & $0.31 \pm 0.05$ \\
& $12.7 \pm 0.82$ & $0.63 \pm 0.06$ & $0.30 \pm 0.05$ \\
120 & $11.4 \pm 0.80$ & $0.57 \pm 0.06$ & $0.27 \pm 0.04$ \\
149 & $8.6 \pm 0.80$ & $0.43 \pm 0.05$ & $0.20 \pm 0.03$ \\
\hline
\end{tabular}




\section{Acknowledgements}

It is a pleasure to acknowledge fruitful discussions with J.-P. Blaizot, K.J. Eskola, D. Kharzeev, P.L. McGaughey, A. Morsch, P.V. Ruuskanen, H. Satz, J. Schukraft, G. Schuler, K. Sridhar, C.Y. Wong and with my colleagues from the NA38, NA51 and NA50 collaborations. Very special thanks are due to Dima, Helmut and Pat.

\section{References}

1. T. Matsui and H. Satz, Phys. Lett. B178 (1986) 416.

2. A. Bussiere et al. (NA38 Coll.), Z. Phys. C38 (1988) 117 (QM '87).

3. C. Baglin et al. (NA38 Coll.), Phys. Lett. B255 (1991) 459.

4. J. Badier et al. (NA3 Coll.), Z. Phys. C20 (1983) 101.

5. C. Baglin et al. (NA38 Coll.), Phys. Lett. B251 (1990) 472.

6. See, for example, the contribution of K. Sridhar to the "31st Rencontres de Moriond: QCD and High Energy Hadronic Interactions", March 1996 (hep-ph/9606252).

7. A. Sansoni et al. (CDF Coll.), these proceedings (FERMILAB-CONF-96-221-E).

8. E. Braaten, these proceedings (hep-ph/9608370).

E. Braaten, S. Fleming and T.C. Yuan, OHSTPY-HEP-T-96001 (hep-ph/9602374).

9. D. Kharzeev and H. Satz, Phys. Lett. B366 (1996) 316 (hep-ph/9508276).

10. C. Gerschel and J. Hüfner, Z. Phys. C56 (1992) 171.

11. D. Kharzeev and H. Satz, Phys. Lett. B334 (1994) 155.

12. M. Gonin et al. (NA50 Coll.), these proceedings.

13. C. Lourenço et al. (NA38 Coll.), Nucl. Phys. A566 (1994) 77c (QM '93).

E. Scomparin et al. (NA50 Coll.), these proceedings.

14. P.L. McGaughey et al. (E772 Coll.), Phys. Rev. D50 (1994) 3038.

15. G. Moreno et al. (E605 Coll.), Phys. Rev. D43 (1991) 2815.

16. J. Badier et al. (NA3 Coll.), Z. Phys. C26 (1984) 489.

17. http://durpdg.dur.ac.uk/scripts/help.csh/REAC/EXP/LISTREACEXP

18. P.L. McGaughey, these proceedings and references therein.

19. W.L. van Neerven, Int. J. Mod. Phys. A10 (1995) 2921.

S. Gavin et al. (Hard Probe Coll.), Int. J. Mod. Phys. A10 (1995) 2961.

20. A. Baldit et al. (NA51 Coll.), Phys. Lett. B332 (1994) 244.

21. P. Amaudruz et al. (NMC Coll.), Phys. Rev. Lett. 66 (1991) 2712.

22. D.M. Alde et al. (E772 Coll.), Phys. Rev. Lett. 66 (1991) 133.

23. M.H. Schub et al. (E789 Coll.), Phys. Rev. D52 (1995) 1307.

24. C. Lourenço et al. (NA38/50 Coll.), Proc. of the Int. Europhysics Conf. on HEP, Brussels 1995, p. 363 (CERN-PRE-95-001).

25. H.D. Snyder et al. (E288 Coll.), Phys. Rev. Lett. 36 (1976) 1415.

A.G. Clark et al., Nucl. Phys. B142 (1978) 29.

L. Antoniazzi et al. (E705 Coll.), Phys. Rev. D46 (1992) 4828.

M.H. Schub et al. (E789 Coll.), Phys. Rev. D52 (1995) 1307.

T. Alexopoulos et al. (E771 Coll.), Phys. Lett. B374 (1996) 271.

26. P.L. Frabetti et al. (E687 Coll.), "J/ $\psi$ and $\psi$ ' Photoproduction in E687", comm. at the Int. Europhysics Conf. on HEP, Brussels 1995, (EPS0705), and ref.s therein.

27. D. Kharzeev and H. Satz, Phys. Lett. B356 (1995) 365. 